\title{
YAP/TAZ for cancer therapy: Opportunities and challenges (Review)
}

\author{
LIWEN GUO and LISONG TENG
}

\author{
Department of Surgical Oncology, The 1st Affiliated Hospital, School of Medicine, \\ Zhejiang University, Hangzhou, Zhejiang 310003, P.R. China
}

Received December 2, 2014; Accepted January 23, 2015

DOI: 10.3892/ijo.2015.2877

\begin{abstract}
YAP (Yes-associated protein) and its paralog TAZ (transcriptional co-activator with PDZ-binding motif) are the main downstream effectors of the Hippo signaling pathway. This pathway is an evolutionally conserved signal cascade, which plays pivotal roles in organ size control and tumorigenesis from Drosophila to mammals. Functionally, when the Hippo pathway is activated, YAP and TAZ will be sequestered in the cytoplasm and degraded. Conversely, when the Hippo pathway is deactivated, YAP and TAZ will translocate into nucleus and promote transcription of downstream genes by forming complexes with transcription factors, such as transcriptional enhancer factors (TEF; also referred to as TEAD), runt-domain transcription factors (Runx) and others. Most of these transcription factors belong to growth promoting or apoptosis-inhibition genes. It has been reported that the deactivation of the Hippo pathway, as well as upregulation of YAP and TAZ was observed in many human cancers with a high frequency, which suggests that the Hippo pathway may be a potent target for developing anticancer drugs. In this review, we provide an overview of the Hippo pathway and summarize recent advances with respect to the role of YAP and TAZ in Hippo signaling pathway and cancer development. Furthermore, we describe the opportunities and challenges for exploit YAP and TAZ as potential therapeutic targets in cancer.
\end{abstract}

\section{Contents}

1. Introduction

2. The Hippo signaling pathway in mammals

3. Similarities and differences between YAP and TAZ in structure and function

4. Transcriptional regulation by YAP/TAZ: partners and targets

Correspondence to: Professor Lisong Teng, Department of Surgical Oncology, The 1st Affiliated Hospital, School of Medicine, Zhejiang University, 79 Qingchun Road,Hangzhou, Zhejiang 310003, P.R. China E-mail: lsteng@zju.edu.cn

Key words: Hippo pathway, YAP, TAZ, cancer, therapeutic target
5. Mechanisms of YAP/TAZ inhibition by the Hippo pathway

6. YAP/TAZ in human cancers

7. YAP/TAZ as potent therapeutic targets in cancer

8. Perspectives

\section{Introduction}

The Hippo signal pathway was first discovered by mosaic genetic screens on Drosophila for mutants exhibiting tissue overgrowth in the $90 \mathrm{~s}(1,2)$. This signal pathway plays pivotal roles in organ growth control, stem cell function, regeneration and tumor suppression $(3,4)$. Wart (Wts) was identified as the first component of the Drosophila Hippo pathway, which encodes a protein kinase of the nuclear Dbf-2-related (NDR) family and plays an important role in controlling cell growth and shape as well as proliferation (5). Subsequent studies uncovered several other Hippo pathway components, including scaffold protein Salvador (Sav), Hippo (Hpo) and Mats in the following years (6). These four proteins formed the center part of the Drosophila Hippo pathway, in which Hpo, in association with Sav, phosphorylate and activate Wts and the adopter protein Mats. Therefore, the Hippo pathway is also known as the Salvador-Warts-Hippo pathway (7). Wts/Mats is a protein kinase complex, thus, it functions through its substrates. By protein interaction screens using Wts as bait, Huang et al identified Yorkie (Yki) as the major substrate of the Wts/Mats complex in the Drosophila (8).

Inactivation of Yki through phosphorylation or mutation caused tissue atrophy, while over-expression of Yki caused massive tissue overgrowth, phenocopying loss of Hpo, Sav or Wts function. Functionally, unphosphorylated Yki can translocate into the nucleus and drive growth-promoting related gene expression, such as cell cycle regulator cyclin E, cell-death inhibitor Diap1, and bantam microRNA $(9,10)$. Thus the localization and phosphorylation of Yki are often taken as a measure of the activity of the Hippo pathway (11). The Hippo pathway is deregulated with a high frequency in many human cancers, which confirmed the Hippo signaling pathway is closely related to the development of cancers (12). Therefore, the discovery of Hippo pathway has generated excitement in both basic and clinical research because targeting the Hippo pathway provide us with new insight into development of anticancer drugs (13). 
Tight junction

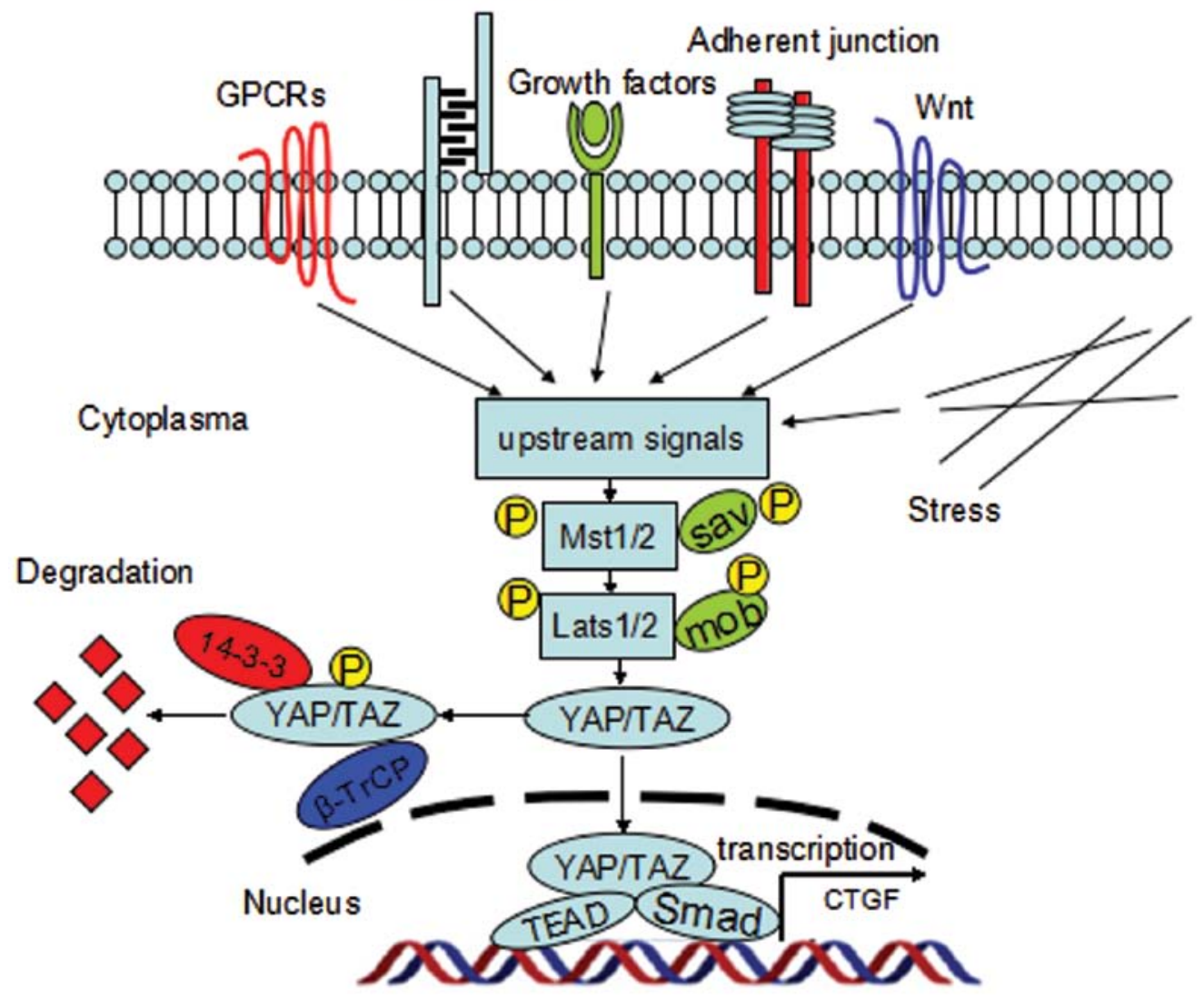

Figure 1. The mammalian Hippo signaling pathway. Upstream signals include the GPCR signaling, Wnt signaling, mechanical stress, tight junctions, adherent junctions, as well as some soluble growth factors. These signals regulate the phosphorylation of Mst1/2 and Lats1/2 kinases. Lats1/2 phosphorylates YAP/TAZ on HXRXXS motifs; and phosphorylation of YAP/TAZ creates a 14-3-3 binding site, which promotes cytoplasmic localization of YAP/TAZ, resulting in YAP/ TAZ cytoplasmic retention and degradation. When YAP/TAZ are dephosphorylated, they enter the nucleus, and act as transcription coactivators by forming complexes with various transcriptional factors such as TEAD, SMAD, to coordinate pro-proliferating and anti-apoptotic programs.

Herein, we provide an overview of the Hippo pathway, with an emphasis on the mammalian system, and summarize the current understanding of the biological functions of YAP and TAZ, and discuss the opportunities and challenges for therapeutic intervention.

\section{The Hippo signaling pathway in mammals}

The Hippo signaling pathway is an evolutionarily conserved pathway; hence, most components of the Drosophila Hippo pathway have mammalian homologues (Table I). For instance, Mst1/2, Sav1, Lats1/2, Mob1 in mammalian are homologues of Hpo, Sav, Wts and Mats, respectively; both YAP and TAZ are homologues of Yki. Similar to the Drosophila Hippo pathway, the center part of the mammalian Hippo pathway is also a protein kinase cascade. Once the upstream activation signals are received, the downstream Mst1/2 kinase phosphorylate and activate the Lats $1 / 2$ kinases and MOB 1 cofactor with the help of SAV1. Then the activated Lats1/2 kinases phosphorylate and inactivate YAP/TAZ transcription co-activators by promoting their cytoplasmic localization and degradation. Conversely, unphosphorylated YAP/TAZ will translocated and accumulated in the nucleus and form complexes with transcription factors, consequently inducing the expression of targeted genes, such as connective tissue growth factor
(CTGF), cysteine-rich 61 (Cyr61), and fibroblast growth factor (FGF1) (14) (Fig. 1).

The upstream signals that regulate the Hippo pathway have not been well delineated, but it consist of a large network of proteins: including a variety of kinases, such as Src, protein kinase A (PKA) (15); some soluble factors, such as amphiregulin (ARGE) (14); cell adhesion and cell junction proteins, such as Echinoid, cadherin-catenin complex $(16,17)$; cell polarity proteins, such as the crumbs complex, scribble (18); other signaling pathways, such as the G-protein-coupled receptor (GPCR) signaling, Wnt and PI3K pathways (19-21); and the state of the actin cytoskeleton $(22,23)$. However, how these proteins impact on YAP/TAZ is not entirely clear. According to the existing experimental conclusions, in addition to regulating YAP/TAZ phosphorylation; these upstream factors also regulate YAP/TAZ nuclear localization through physical interaction.

\section{Similarities and differences between YAP and TAZ in structure and function}

YAP was first identified as a proline-rich phosphoprotein bound to the SH3 domain of Yes and Src protein tyrosine kinases (24). The human YAP gene, located at 11q13, encodes a $65 \mathrm{kDa}$ protein. Eight isoforms of YAP protein have been 
Table I. Hippo signaling pathway components in mammalian and homologues in Drosophila.

\begin{tabular}{|c|c|c|c|}
\hline $\begin{array}{l}\text { Mammalian } \\
\text { proteins }\end{array}$ & $\begin{array}{l}\text { Drosophila } \\
\text { homologues }\end{array}$ & $\begin{array}{l}\text { Conserved domains } \\
\text { and motifs }\end{array}$ & Main functions \\
\hline
\end{tabular}

\section{Upstream modulators}

Fat4 Fat

Dchs $1 / 2$

Fjx 1

Ck1

ZDHHC9/14/18

Patj

FRMD6, Ex1

PTPN14

NF2

Kibra

Lgl1,2

Crb1-3

\section{DLG1-4}

\section{SCRIB}

Rassf1-6

Ajuba

Mpp5

Amot, AmotL

TAO1-3

E-cadherin

$\alpha$-catenin

Core components

$\begin{array}{ll}\text { Mst1/2 } & \text { Hpo } \\ \text { Lats1/2 } & \text { Wts } \\ \text { YAP/TAZ } & \text { Yki }\end{array}$

Sav1 Sav

Mob1/2

\section{Mats}

Tao

E-cadherin

$\alpha$-catenin

$\begin{array}{ll}\text { Hpo } & \text { Kinase domain, SARAH domain } \\ \text { Wts } & \text { Kinase domain } \\ \text { Yki } & \text { WW domains, 14-3-3 BM, } \\ & \text { TEAD-BM PDZ-BM } \\ \text { Sav } & \text { FERM domain, WW domains, } \\ & \text { SARAH domain } \\ \text { Mats } & \text { MOB domain }\end{array}$

Downstream mediators

$\begin{array}{ll}\text { TEAD1-4 } & \text { Sd } \\ \text { Smads } & \text { MAD } \\ \text { TSHZ1-3 } & \text { Tsh } \\ \text { Wbp2 } & \text { Wbp2 }\end{array}$

EGF-like repeats, laminin

A-G domain

Cadherin repeated domain

Golgi Ser/thr kinase

ser/thr kinase domain

DHHC domain

Ribosomal protein L27, PDZ domain

FERM domain

FERM domain

FERM domain

WW domains, C2 domain

LLGL2 domain

EGF domains, laminin A-G domains, transmembrane domain

Three PDZ domains,

SH3 domain, GUK domain

LRR domains, PDZ domains

RAS association domain,

SARAH domain

LIM domains

Ribosomal protein L27, PDZ domain, SH3 domain, GUK domain

CC domain, PDZ-BM

Kinase domain

cadherin domain

VH1-VH3 domains

Kinase domain, SARAH domain

WW domains, 14-3-3 BM,

TEAD-BM PDZ-BM

FERM domain, WW domains,

MOB domain

TEAD DNA-binding domain, vestigial binding domain

MH1 domain

$\mathrm{C} 2 \mathrm{H} 2$ type zinc-finger domain

WW domain, GRAM domain
Adhesion molecule and/or signaling receptor

Cell-adhesion protein

Legs, wings and eyes development

Protein kinase

DHHC palmitoyltransferase

Adaptor protein

Adaptor protein

Phosphates

Adaptor protein

Adaptor protein

WD40 scaffold protein

Transmembrane

receptor

Adaptor protein

Adaptor protein

Adaptor protein

Adaptor protein

Adaptor protein

Adaptor protein

Serine/threonine kinase

Transmembrane receptor

Adaptor protein

Serine/threonine kinase

Serine/threonine kinase

Transcriptional

co-activator

Adaptor protein

Adaptor protein

Transcription factor

Transcription factor

Zn-finger transcription factor

Cofactor

Fat, FAT atypical cadherin 4; EGF, epidermal growth factor; CK1, casein kinase 1; AMOT, angiomotin; AMOTL, angiomotin-like protein; CRB1, Crumbs homolog 1; Dco, discs overgrown; App, approximated; Dlg, discs large; Fjx1, Four jointed box 1; Ex, Expanded; FERM, 4.1, ezrin, radixin and moesin; Lgl, lethal giant larvae; GUK, guanylyl kinase; Mer, Merlin; MPP5, membrane protein, palmitoylated 5; MUPP1, multiple PDZ domain protein 1; NF2, neurofibromin 2; PATJ, PALS1-associated tight junction protein; PTPN14, protein tyrosine phosphatase, non-receptor type 14; RASSF, RAS association domain-containing family protein; SARAH, Sav-Rassf-Hpo domain; SCF, SKP1cullin-1-F-box complex; SCRIB, Scribble; Sd, Scalloped; Sdt, Stardust; TAO, thousand and one amino acid protein; VH1, vinculin head 1; MH1, Mad Homology 1; ZDHHC9/14/18 (zinc finger, DHHC-type containing 9/14/18); LLGL2, lethal giant larvae homolog 2; Hpo, Hippo; KIBRA, kidney and brain protein; LATS1, large tumour suppressor homolog 1; Wts, Warts; YAP, Yes-associated protein; Yki, Yorkie; TAZ, transcriptional co-activator with PDZ-binding motif; MOB1A, MOB kinase activator 1A; MST, mammalian STE20-like protein kinase; SAV1, Salvador homolog 1; GRAM, Rab-like GTPase activators and myotubularins domain; TEAD, TEA domain-containing sequence-specific transcription factor; WBP2, WW domain-binding protein 2; MAD, Mothers against dpp; TSHZ1-3, teashirt zinc finger homeobox 1-3. 

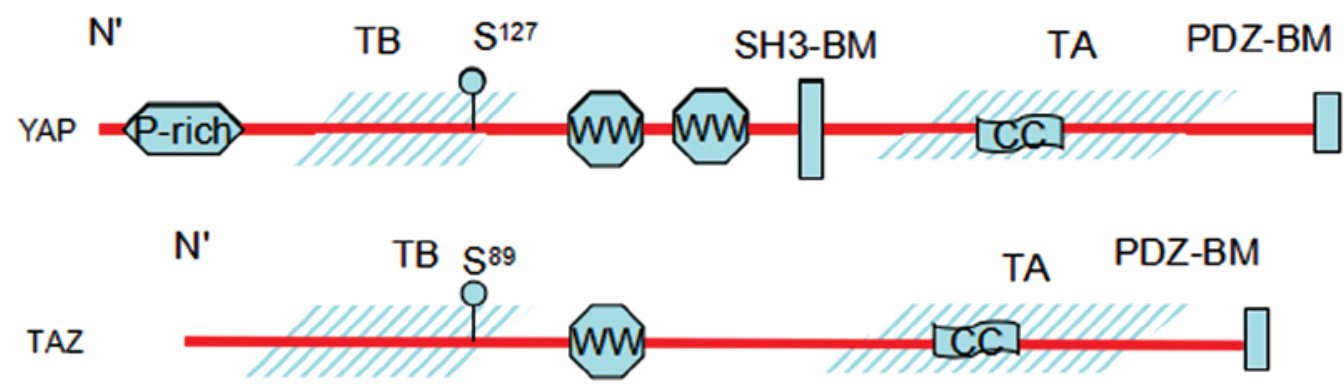

Figure 2. A schematic view of the structures of YAP and TAZ. YAP has a proline-rich (P-rich) region at the N-terminal, a TEAD family transcription factor binding domain (TB), two tandem WW domains in the middle followed by an Src homology domain 3 binding motif (SH3 BM), a coiled-coiled motif (CC) in the transactivation domain (TA) and a PDZ-binding motif (PDZ-BM) in the C-terminal. TAZ has a similar domain organization with YAP, but lacks the proline-rich domain, the second WW domain, and the SH3-binding motif.

identified in humans to date, which are differed by either an alteration in the activation domain or a loss of WW domain $(25,26)$. YAP mRNA is not only ubiquitously expressed in a broad range of tissues; but also expressed in the whole developmental process (27). YAP gene is amplified in various human cancers. TAZ, also known as WWTR1 (WW-domain containing transcriptional regulator 1), was first identified in 2000 as a 14-3-3 binding protein. The human TAZ gene, located at 3q23-q24, encodes a $43 \mathrm{kDa}$ protein. Similarly, TAZ is expressed in various tissues, except thymus and peripheral blood leukocytes and amplified in various human cancers (28).

Structurally, YAP and TAZ share nearly half of the overall amino acid sequence and have very similar topology. YAP protein consists of 488 amino acids, has a TEAD-binding region (TB), two WW domains, which consist of two conserved tryptophan (W) residues separated by 20-23 amino acids, an SH3-binding motif, a coiled-coil domain, a transcription activation domain, an N-terminal proline-rich domain, and a C-terminal PDZ-binding motif. TAZ protein consists of 400 amino acids, has a similar domain organization with YAP, although it lacks the second WW domain, the SH3-binding motif, and the proline-rich domain (Fig. 2).

Biochemically, the WW domains of the YAP and TAZ have been shown to interact with PPXY motifs (proline/ proline/any amino acid/tyrosine) of some transcriptional factors. The TB domain recognizes the TEAD family of transcription factors and activates target gene expression, whereas the 14-3-3 binding motif is crucial to the regulation of YAP and TAZ. The PDZ-binding motif is required for binding with PDZ domain, which are found in many transmembrane or cytoskeleton associated proteins. The PDZ-binding domains appear to direct YAP and TAZ localization $(29,30)$.

At the functional level, both of the YAP and TAZ are serving as transcriptional co-activator and share some transcription factor partners, such as TEAD and Runx. Both of them promote cell proliferation, increases cell migration and invasion. However, both of them have their unique target transcription factors, such as ErbB4, p73 for YAP and PPAR $\gamma$, Pax3, TBX5, TTF-1 for TAZ, which may contribute to the differential functions of YAP to TAZ. Moreover, gene knockout mouse models demonstrated the different physiological functions between YAP and TAZ: Knockout of the YAP gene in mice leads to early developmental arrest, suggesting an essential role in development. However, TAZ knockout mice are viable, although they have defects in the kidneys and lungs.

\section{Transcriptional regulation by YAP/TAZ: partners and targets}

Although YAP and TAZ are the main downstream effectors of the Hippo pathway in the mammals, YAP and TAZ do not have DNA binding domains. Alternatively, once they localize to the nucleus, they interact with transcription factor partners and function as transcription co-activators. YAP and TAZ share some transcription factor partners, such as TEAD and Runx proteins.

The mammalian genome contains four highly homologous members of the TEAD protein family (TEAD1-4), which are the major targets of the YAP and TAZ in regulation of cell contact inhibition, epithelial-mesenchymal transition (EMT), oncogenic transformation and apoptosis inhibition (31). The interaction of the TEAD with the TB domains of YAP and TAZ can activate downstream gene transcripts, such as CTGF, and Cyr61. Disrupting the binding of YAP or TAZ to TEAD by genetic approach will abolish their ability to promote cell proliferation and tumorigenesis (32-34). For instance, a mutation of TEAD1 in $\mathrm{Y} 421 \mathrm{H}$ leads to the human genetic disease Sveinsson's chorioretinal atrophy, which is characterized by helicoid peripapillary chorioretinal degeneration or atrophia areata (35). Besides the TB domains, the WW domains of the YAP and TAZ have been shown to interact with PPXY motifs of some transcriptional factors. Runx transcription factors consist of 3 members (Runx1, Runx2 and Runx3), all have a PPXY motif and are involved in carcinogenesis and cancer metastasis. Both YAP and TAZ can bind to and potentiate Runx activity through WW-PPXY interaction $(36,37)$.

In addition to the above binding modes, YAP and TAZ can regulate expression of some genes through other means. TAZ increases the transcriptional activity of thyroid transcription factor-1(TTF-1) on the surfactant protein $\mathrm{C}$ promoter by directly interacting with the N-terminal domain of TTF-1 (38). T-box 5 gene (TBX5) belongs to the T-box family of transcription factors that plays an essential role in cardiac and limb development. YAP and TAZ can stimulate TBX5 transcription by interaction with multiple domains including carboxylterminal structure of the TBX5 (39). Through the coiled-coil region, YAP and TAZ are able to interact with $\mathrm{Smad} 2 / 3 / 4$ and 
consequently activate transforming growth factor- $\beta$ target gene expression and maintain human embryonic stem cell self-renewal (40).

However, both YAP and TAZ have their own specific binding partners, although they are very similar in their structure. For instance, TAZ inhibits the transcriptional activation of PPAR $\gamma$, which is critical for adipocyte differentiation, through the interaction of WW domain with PPXY motif. This inhibition prevented mesenchymal stem cells from differentiating into adipocytes. However, although YAP has WW domains, it is unable to interact with PPAR $\gamma$ (41). TAZ, but not YAP, interacts with the PPXY motif of the Kruppel-like factor 5 (KLF5) through the WW domain and protects KLF5 from WWP1mediated ubiquitination and degradation, thereby, promotes breast cell proliferation and tumorigenesis (42). On the other hand, YAP also has its specific binding partners, such as p73 and ErBb4. Strano et al demonstrated the WW domain of YAP interacts with PPXY motif of the p73, consequently enhancing p73 transcription activity and accelerating programmed cell death (43). Komuro et al reported YAP activates ErbB4dependent transcription by forming the YAP-ErbB4 complex, which is mediated by the WW domain of YAP and the PPXY motif of ErbB-4 (44).

\section{Mechanisms of YAP/TAZ inhibition by the Hippo pathway}

Phosphorylation of YAP $\mathrm{S}^{127}$ and the corresponding site of TAZ by Lats1/2 promote 14-3-3 protein binding resulting in YAP/TAZ cytoplasm retention and functional inhibition, because they were separated from their nuclear binding partners. Mutation of YAP $\mathrm{S}^{127}$ or equivalent residues in TAZ abrogated the 14-3-3 protein interaction thus enhancing their function as transcriptional co-activators (45).

In addition to promoting YAP/TAZ translocation, phosphorylation by Lats1/2 also suppress their activities by inducing proteasomal degradation. The Lats1/2 phosphorylate the C-terminal phosphodegrons in YAP/TAZ, primed them for subsequent phosphorylation by casein kinase 1, eventually resulting in the recruitment of the $\mathrm{SCF}^{\beta-\operatorname{TrCP}}$ ligase, thereby leading to poly-ubiquitylation and degradation of YAP/TAZ (46). The N-terminal phosphodegron of TAZ can be phosphorylated by GSK3 via PI3K signaling, resulting in $\mathrm{SCF}^{\beta-\operatorname{TrCP}} \mathrm{E} 3$ ubiquitin ligase-mediated proteolysis of TAZ (47). Disruption of the interaction between YAP/TAZ and $\beta$-TrCP by mutation will stabilize TAZ/YAP and promote their function.

YAP/TAZ can also be inhibited in a protein-protein interaction manner, the PPXY motifs on Lats1/2, AMOT, and PTPN14 have been shown to directly interact with WW domains on YAP/TAZ and thereby convey inhibitory signals by sequestering YAP/TAZ in the cytosol or junctional compartments $(48,49)$. Through its PDZ domain, junctional protein zona occludens-2 (ZO-2) can directly bind with YAP, consequently promoting YAP translocation to the nucleus $(50,51)$.

\section{YAP/TAZ in human cancers}

Numerous studies show that overexpression of YAP/TAZ can induce EMT, inhibiting apoptosis and increase the number of cancer stem cells in vitro (52-54). Knockdown of YAP/ TAZ expression reduced cancer cell migration, invasion and anchorage-independent growth in soft agar, as well as tumorigenesis in nude mice (54-56). The protein level of TAZ is correlated with the invasiveness of breast cancer cells (54). In transgenic mouse models, over-expression of YAP/TAZ or attenuation of Hippo signaling is sufficient to promote tumor formation $(45,57,58)$.

Indeed, the levels and nuclear localization of YAP/TAZ are elevated in many human cancers, such as carcinomas of the lung, thyroid, ovarian, colorectal, prostate, pancreas, esophagus, liver, mammary gland $(12,59,60)$, and positively correlated with poorly differentiated tumors (53). Higher YAP/TAZ protein levels are associated with shorter patient overall survival (61-63). Xie et al reported TAZ protein was expressed in $66.8 \%(121 / 181)$ of non-small cell lung cancer patients (NSCLC) cases and significantly associated with poorer differentiation and short survival (64). Yue et al reported TAZ protein expression was positive in 113 out of $146(77.4 \%)$ gastric cancer samples and especially higher expressed in signet ring cell carcinoma (65). The studies of Yuen et al demonstrated that the mRNA level of TAZ is a prognostic marker in colon cancer progression (61). Wang et al reported YAP was overexpressed in $66.3 \%$ (61/92) specimens of NSCLC and significantly correlated with p-TNM stage and lymph node metastasis (62). Lam-Himlin et al found that YAP expression in the cytoplasm and nucleus is significantly increased in high-grade dysplasia and adenocarcinoma of the esophagus as well as gastric adenocarcinoma and metastatic gastric disease (66).

However, some researchers suggest YAP might function as a tumor suppressor. Knockdown of YAP in breast cell lines suppressed anoikis, increased migration and invasiveness, enhanced tumor growth in nude mice, and decreased Taxol responsiveness $(67,68)$. Overexpression of YAP can induce p19 cell apoptosis by increase p73 alpha transcriptional activity (39). YAP is also a critical component of c-Jun-mediated induction of apoptosis $(43,69)$. In contrast, TAZ have not been found to have tumor suppressor function. These research studies suggest the possibility that YAP may have dual roles, as tumor promoter or tumor suppressor, which may depend on the cell context.

\section{YAP/TAZ as potent therapeutic targets in cancer}

Considerable number of studies show that the Hippo pathway plays a key role in tumorigenesis. Downregulation of the Hippo pathway activity not only promotes cancer cells growth, but also makes cancer cells resistant to some chemotherapy drugs (70). These founding raised the possibility that therapeutic intervention of Hippo signaling may improve current treatment strategies. We focus on small molecules that are designed to target Hippo signaling itself by manipulating core components of the Hippo pathway (Table II).

Targeting YAP/TAZ-TEAD interaction or upstream kinases. In many cases, the tumorigenesis potential of YAP and TAZ require association to TEAD proteins. Blocking the formation of the YAP/TAZ-TEAD complex will abolish the transforming ability of YAP and TAZ in vitro $(33,34)$; by screening 
Table II. Small-molecule modulators of the Hippo pathway.

\begin{tabular}{|c|c|c|}
\hline Compounds & Targets and effects & Refs. \\
\hline 17-mer & YAP-like peptides, abolish YAP-TEAD interaction & (73) \\
\hline Verteporfin (VP) & Binding to YAP, thus inhibit YAP-TEAD interaction & $(71)$ \\
\hline $9 \mathrm{E} 1$ & $\begin{array}{l}\text { Mst1 inhibitor, inhibit Mst1 kinase activity, } \\
\text { thus impair YAP/TAZ degradation }\end{array}$ & (74) \\
\hline $\begin{array}{l}\text { LPA, S1P, } \\
\text { Thrombin, } \\
\text { Epinephrin, } \\
\text { Glucagon }\end{array}$ & $\begin{array}{l}\text { Targeting GPCR signaling pathway, act through } \\
\text { GPCRs to inhibit the Lats } 1 / 2 \text { kinases, } \\
\text { thereby activating YAP/TAZ }\end{array}$ & $(20,82,83)$ \\
\hline Butamine & $\begin{array}{l}\text { G protein-coupled } \beta \text {-adrenergic receptor agonist, } \\
\text { prevent YAP nuclear accumulation }\end{array}$ & $(86)$ \\
\hline Dasatinib & $\begin{array}{l}\text { SRC family inhibitor, inhibits YES } 1 \text {, resulted in } \\
\text { inactivation of the YAP1- } \beta \text {-catenin-TBX5 complex, and } \\
\text { thus impaired the proliferation of } \beta \text {-catenin active cells }\end{array}$ & $(75,76)$ \\
\hline $\begin{array}{l}\text { Atrunculin A/B, } \\
\text { Cytochalasin D } \\
\text { Blebbistatin }\end{array}$ & $\begin{array}{l}\text { F-actin destabilizers, inhibit YAP nuclear localization as } \\
\text { well as YAP and TEAD activity in various cell lines; } \\
\text { promoting YAP/TAZ nuclear translocation }\end{array}$ & $(77-80)$ \\
\hline Y27632 & $\begin{array}{l}\text { RHO-ROCK signaling inhibitor, prevent YAP } \\
\text { from phosphorylating by Lats } 1 / 2 \text {, thus promote YAP } \\
\text { nuclear accumulation }\end{array}$ & $(81)$ \\
\hline $\begin{array}{l}\text { XAV939, G007-LK, } \\
\text { G244-LM }\end{array}$ & $\begin{array}{l}\text { Tankyrase inhibitors, restore the activity of Axin by } \\
\text { preventing PAR-dependent Axin degradation, led to } \\
\text { YAP/TAZ degradation via the } \beta \text {-catenin destruction complex }\end{array}$ & $(88,89)$ \\
\hline $\begin{array}{l}\text { Statins } \\
\text { Bisphosphonates }\end{array}$ & $\begin{array}{l}\text { SREBP/mevalonate pathway inhibitors, inhibit YAP/TAZ } \\
\text { nuclear localization and activation through metabolic control }\end{array}$ & $(90)$ \\
\hline
\end{tabular}

of a small-molecule library, several porphyrin compounds have been found out to bind to YAP, and subsequently inhibit the interaction of YAP and TEAD. One of these porphyrin compounds, verteporfin (VP), is already used for photodynamic therapy of macular degeneration in the clinic; repeated administration of VP was effective in delaying tumor progression and suppressing liver overgrowth in a liver cancer mouse model, without overt adverse effects in other organs (71). Vestigial-like protein 4 (VGLL4) is a natural antagonist of YAP, because its TDU region has a structure similar to TB domains of YAP, thus can competitively inhibit the interaction of YAP-TEAD. These findings led to the generation of peptide-based YAP inhibitors (72,73). In addition, small-molecule inhibitors of the upstream kinases, such as Mst1 inhibitor 9E1, would upregulate YAP and TAZ function (74). Src family kinase inhibitor, Dasatinib, inhibits kinase YES1, resulting in inactivation of the YAP1- $\beta$-catenin-TBX5 complex, and thus impaired the proliferation of $\beta$-catenin active cells $(75,76)$.

Targeting F-actin. F-actin can inhibit Hippo pathway activity, thereby promoting YAP/TAZ nuclear translocation, which leads to upregulation of proliferation and survival genes (77-79). Therefore, it is reasonable to speculate that drugs targeting F-actin may be effective in control of tumor growth. The F-actin destabilizers, such as cytochalasin D, latrunculin A/B, together with RHO kinase inhibitor Y27632 and the non-muscle myosin II inhibitor blebbistatin all cause nuclear export of YAP and TAZ $(80,81)$. However, it is worth noting that the actin cytoskeleton is important for many basic cellular functions. Therefore, choosing a proper dosage of F-actin inhibitors would improve the pharmacotherapy efficacy and reduce the toxic side effects.

Targeting GPCR signaling pathway. GPCR represent one of the largest gene families in the human genome and regulate a wide array of physiological functions. Yu et al reported GPCR signaling can either activate or inhibit the activities of YAP/TAZ depending on the coupled G protein (20). Serum-borne lysophosphatidic acid (LPA), sphingosine 1-phosphophate (S1P), thrombin and protease-activated receptor agonist peptides act through $\mathrm{G}_{12 / 13}$ coupled receptors to inhibit the Lats1/2 kinases, thereby activating YAP/TAZ (82); while adrenaline, glucagon and dihydrexidine activate Gas coupled receptors, result in enhanced YAP phosphorylation and inactivation (83). Therefore, development of antibodies or small-molecule agonists mimicking LPA or S1P stimulation may be an effective therapeutic option. Many of these drugs have been developed and, such as the S1P-blocking antibody Sphingomab, Phosphatase-resistant LPA analogs were evaluated in clinic trails $(84,85)$. By cell-based method of screening, another $\mathrm{G}$ protein-coupled $\beta$-adrenergic receptor agonist, dobutamine, was identified and shown to be effective in preventing 
YAP nuclear accumulation and YAP-mediated transcriptional activation. Of note, the effect of dobutamine is independent of the Hippo pathway, which means it is possible to develop lower side effect Hippo-independent YAP inhibitors $(20,86)$.

Targeting Wnt/ $\beta$-catenin signaling pathway. It was reported that YAP/TAZ serve as downstream elements of the Wnt/ $/ \beta$-catenin signaling pathway with independent of the Hippo signal pathway. Once Wnt signaling was turned on, $\beta$-catenin detached from the destruction complex, formed by APC, Axin and GSK3, resulting in inhibition of YAP/TAZ degradation then leading to YAP/TAZ activation $(19,87)$. Therefore, targeting the Wnt pathway by restoring the integrity of the destruction complex would effectively inhibit YAP/ TAZ. Some tankyrase inhibitors, such as XAV939, G007-LK and G244-LM can restore the integrity of the destruction complex by increasing the Axin activity, leading to YAP/TAZ degradation via the $\beta$-catenin destruction complex $(88,89)$.

Targeting metabolic cues. It was recently reported that YAP/ TAZ activity is controlled metabolically via the SREBP/ mevalonate pathway. Functionally, mevalonate is a precursor for geranylgeranyl diphosphate (GGPP) that, in turn, promotes Rho GTPase membrane localization and activity, thus leading to YAP/TAZ nuclear localization and activation. Inhibition of this pathway by means of statins, bisphosphonates or geranylgeranyl transferase inhibitors attenuates YAP/TAZ biological activities (90). The significance of this finding is that it opened up new ideas for designing anti-YAP/TAZ drugs.

\section{Perspectives}

The main reasons why the Hippo signaling pathway gained so much attention since it was delineated are the crucial roles in organ size control, cell differentiation, as well as tumorigenesis. The Hippo pathway activity is associated with cancer cell's proliferative potential, multi-drug resistance, EMT and metastasis. In the past dozen years, our understanding of the Hippo signaling pathway, in both Drosophila and mammalian, has largely increased; the kinase cascade and the main downstream effectors of this pathway were basically defined. However, there are still many questions to be answered: i) although a wide range of upstream signals have been identified, the details mechanism by which these upstream regulators integrated to impact on the core components is not yet fully understood. ii) Some components also interplay with other pathways; the in-depth mechanisms need to be uncovered. iii) YAP and TAZ have similar structure and both serve as downstream effectors in the Hippo pathway, but they also have distinct functions. Is there some internal relationship between YAP and TAZ? Are there any other transcription factors and target genes mediating YAP/TAZ function? iv) Why does the Hippo pathway vary in terms of cell context? Are there any predictive biomarkers that enable us to select appropriate patients for anti-YAP/TAZ drugs treatment? Answers to these questions would advance our understanding of the Hippo pathway, as well as provide us valuable information for new drug development.

There are numerous potential drug targets identified to modulate the Hippo pathway and some new drugs are under clinical trials. However, a batch of new problems have appeared: most of the targets lack of specificity, such as upstream regulators GPCR, which have broad physiological functions in normal cells. General interference of these targets may cause serious side effects. Directly targeting the core components, such as YAP and TAZ, would improve specificity, but YAP and TAZ also play important roles in organ size control and normal tissue regeneration. It means that long-term whole-body inhibition of either of these proteins may results in serious side effects. It would be ideal to develop effective means to manipulate the Hippo pathway and YAP/TAZ activity in both a tissue specific and transient manner. Undoubtedly, the Hippo pathway and YAP/TAZ may represent valuable targets for cancer therapy, although further research is necessary to definite the upstream regulators, downstream targets, as well as the regulation mechanisms of this pathway.

\section{References}

1. Harvey K and Tapon N: The Salvador-Warts-Hippo pathway an emerging tumour-suppressor network. Nat Rev Cancer 7: 182-191, 2007.

2. Watson KL, Justice RW and Bryant PJ: Drosophila in cancer research: The first fifty tumor suppressor genes. J Cell Sci (Suppl) 18: 19-33, 1994.

3. Halder G and Johnson RL: Hippo signaling: Growth control and beyond. Development 138: 9-22, 2011.

4. Mo JS, Park HW and Guan KL: The Hippo signaling pathway in stem cell biology and cancer. EMBO Rep 15: 642-656, 2014.

5. Justice RW, Zilian O, Woods DF, Noll M and Bryant PJ: The Drosophila tumor suppressor gene warts encodes a homolog of human myotonic dystrophy kinase and is required for the control of cell shape and proliferation. Genes Dev 9: 534-546, 1995.

6. Wu S, Huang J, Dong J and Pan D: Hippo encodes a Ste-20 family protein kinase that restricts cell proliferation and promotes apoptosis in conjunction with salvador and warts. Cell 114: 445-456, 2003.

7. Udan RS, Kango-Singh M, Nolo R, Tao C and Halder G: Hippo promotes proliferation arrest and apoptosis in the Salvador/ Warts pathway. Nat Cell Biol 5: 914-920, 2003.

8. Huang J, Wu S, Barrera J, Matthews K and Pan D: The Hippo signaling pathway coordinately regulates cell proliferation and apoptosis by inactivating Yorkie, the Drosophila Homolog of YAP. Cell 122: 421-434, 2005.

9. Thompson BJ and Cohen SM: The Hippo pathway regulates the bantam microRNA to control cell proliferation and apoptosis in Drosophila. Cell 126: 767-774, 2006.

10. Koontz LM, Liu-Chittenden Y, Yin F, Zheng Y, Yu J, Huang B, Chen Q, Wu S and Pan D: The Hippo effector Yorkie controls normal tissue growth by antagonizing scalloped-mediated default repression. Dev Cell 25: 388-401, 2013.

11. Hong W and Guan KL: The YAP and TAZ transcription co-activators: Key downstream effectors of the mammalian Hippo pathway. Semin Cell Dev Biol 23: 785-793, 2012.

12. Harvey KF, Zhang $X$ and Thomas DM: The Hippo pathway and human cancer. Nat Rev Cancer 13: 246-257, 2013.

13. Stanger BZ: Quit your YAPing: A new target for cancer therapy. Genes Dev 26: 1263-1267, 2012.

14. Wang L, Chen Z, Wang Y, et al: WWTR1 promotes cell proliferation and inhibits apoptosis through cyclin A and CTGF regulation in non-small cell lung cancer. Tumour Biol 35: 463-468, 2014

15. Yu FX, Zhang Y, Park HW, Jewell JL, Chen Q, Deng Y, Pan D, Taylor SS, Lai ZC and Guan KL: Protein kinase A activates the Hippo pathway to modulate cell proliferation and differentiation. Genes Dev 27: 1223-1232, 2013.

16. Kim NG, Koh E, Chen X and Gumbiner BM: E-cadherin mediates contact inhibition of proliferation through Hippo signaling-pathway components. Proc Natl Acad Sci USA 108: 11930-11935, 2011.

17. Yue T, Tian A and Jiang J: The cell adhesion molecule echinoid functions as a tumor suppressor and upstream regulator of the Hippo signaling pathway. Dev Cell 22: 255-267, 2012. 
18. Verghese S, Waghmare I, Kwon H, Hanes K and Kango-Singh M: Scribble acts in the Drosophila fat-hippo pathway to regulate warts activity. PLoS One 7: e47173,2012.

19. Konsavage WM Jr and Yochum GS: Intersection of Hippo/YAP and Wnt/ $\beta$-catenin signaling pathways. Acta Biochim Biophys Sin (Shanghai) 45: 71-79, 2013.

20. Yu FX, Zhao B, Panupinthu N, et al: Regulation of the Hippo-YAP pathway by G-protein-coupled receptor signaling. Cell 150: 780-791, 2012.

21. Tumaneng K, Schlegelmilch K, Russell RC, Yimlamai D, Basnet H, Mahadevan N, Fitamant J, Bardeesy N, Camargo FD and Guan KL: YAP mediates crosstalk between the Hippo and PI(3)K-TOR pathways by suppressing PTEN via miR-29. Nat Cell Biol 14: 1322-1329, 2012.

22. Dai X, She P, Chi F, et al: Phosphorylation of angiomotin by Lats1/2 kinases inhibits F-actin binding, cell migration, and angiogenesis. J Biol Chem 288: 34041-34051, 2013.

23. Hong W: Angiomotin'g YAP into the nucleus for cell proliferation and cancer development. Sci Signal 6: pe27, 2013.

24. Sudol M: Yes-associated protein (YAP65) is a proline-rich phosphoprotein that binds to the SH3 domain of the Yes protooncogene product. Oncogene 9: 2145-2152, 1994.

25. Sudol M, Bork P, Einbond A, Kastury K, Druck T, Negrini M, Huebner K and Lehman D: Characterization of the mammalian YAP (Yes-associated protein) gene and its role in defining a novel protein module, the WW domain. J Biol Chem 270: 14733-14741, 1995.

26. Gaffney CJ, Oka T, Mazack V, et al: Identification, basic characterization and evolutionary analysis of differentially spliced mRNA isoforms of human YAP1 gene. Gene 509: 215-222, 2012.

27. Morin-Kensicki EM, Boone BN, Howell M, Stonebraker JR, Teed J, Alb JG, Magnuson TR, O'Neal W and Milgram SL: Defects in yolk sac vasculogenesis, chorioallantoic fusion, and embryonic axis elongation in mice with targeted disruption of Yap65. Mol Cell Biol 26: 77-87, 2006.

28. Kanai F, Marignani PA, Sarbassova D, et al: TAZ: A novel transcriptional co-activator regulated by interactions with 14-3-3 and PDZ domain proteins. EMBO J 19: 6778-6791, 2000.

29. Oka T and Sudol M: Nuclear localization and pro-apoptotic signaling of YAP2 require intact PDZ-binding motif. Genes Cells 14: 607-615, 2009.

30. Remue E, Meerschaert K, Oka T, Boucherie C, Vandekerckhove J, Sudol M and Gettemans J: TAZ interacts with zonula occludens-1 and -2 proteins in a PDZ-1 dependent manner. FEBS Lett 584 4175-4180, 2010.

31. Sawada A, Kiyonari H, Ukita K, Nishioka N, Imuta $Y$ and Sasaki H: Redundant roles of Tead1 and Tead2 in notochord development and the regulation of cell proliferation and survival Mol Cell Biol 28: 3177-3189, 2008.

32. Lamar JM, Stern P, Liu H, Schindler JW, Jiang ZG and Hynes RO: The Hippo pathway target, YAP, promotes metastasis through its TEAD-interaction domain. Proc Natl Acad Sci USA 109: E2441-E2450, 2012

33. Zhang H, Liu CY, Zha ZY, Zhao B, Yao J, Zhao S, Xiong Y, Lei QY and Guan KL: TEAD transcription factors mediate the function of TAZ in cell growth and epithelial-mesenchymal transition. J Biol Chem 284: 13355-13362, 2009.

34. Zhao B, Ye X, Yu J, et al: TEAD mediates YAP-dependent gene induction and growth control. Genes Dev 22: 1962-1971, 2008 .

35. Fossdal R, Jonasson F, Kristjansdottir GT, Kong A, Stefansson H, Gosh S, Gulcher JR and Stefansson K: A novel TEAD1 mutation is the causative allele in Sveinsson's chorioretinal atrophy (helicoid peripapillary chorioretinal degeneration). Hum Mol Genet 13: 975-981, 2004

36. Yagi R, Chen LF, Shigesada K, Murakami Y and Ito Y: A WW domain-containing yes-associated protein (YAP) is a novel transcriptional co-activator. EMBO J 18: 2551-2562, 1999.

37. Zaidi SK, Sullivan AJ, Medina R, Ito Y, van Wijnen AJ, Stein JL, Lian JB and Stein GS: Tyrosine phosphorylation controls Runx2mediated subnuclear targeting of YAP to repress transcription. EMBO J 23: 790-799, 2004

38. Park KS, Whitsett JA, Di Palma T, Hong JH, Yaffe MB and Zannini M: TAZ interacts with TTF-1 and regulates expression of surfactant protein-C. J Biol Chem 279: 1738417390,2004

39. Murakami M, Nakagawa M, Olson EN and Nakagawa O: A WW domain protein TAZ is a critical coactivator for TBX5, a transcription factor implicated in Holt-Oram syndrome. Proc Natl Acad Sci USA 102: 18034-18039, 2005.
40. Varelas X, Sakuma R, Samavarchi-Tehrani P, Peerani R, Rao BM, Dembowy J, Yaffe MB, Zandstra PW and Wrana JL: TAZ controls Smad nucleocytoplasmic shuttling and regulates human embryonic stem-cell self-renewal. Nat Cell Biol 10 837-848, 2008

41. Hong JH, Hwang ES, McManus MT, et al: TAZ, a transcriptional modulator of mesenchymal stem cell differentiation. Science 309: 1074-1078, 2005

42. Zhao D, Zhi X, Zhou Z and Chen C: TAZ antagonizes the WWP1-mediated KLF5 degradation and promotes breast cell proliferation and tumorigenesis. Carcinogenesis 33: 59-67, 2012.

43. Strano S, Munarriz E, Rossi M, Castagnoli L, Shaul Y, Sacchi A, Oren M, Sudol M, Cesareni G and Blandino G: Physical interaction with Yes-associated protein enhances p73 transcriptional activity. J Biol Chem 276: 15164-15173, 2001.

44. Komuro A, Nagai M, Navin NE and Sudol M: WW domaincontaining protein YAP associates with ErbB-4 and acts as a co-transcriptional activator for the carboxyl-terminal fragment of ErbB-4 that translocates to the nucleus. J Biol Chem 278: 33334-33341, 2003.

45. Dong J, Feldmann G, Huang J, Wu S, Zhang N, Comerford SA, Gayyed MF, Anders RA, Maitra A and Pan D: Elucidation of a universal size-control mechanism in Drosophila and mammals. Cell 130: 1120-1133, 2007.

46. Zhao B, Li L, Tumaneng K, Wang CY and Guan KL: A coordinated phosphorylation by Lats and CK1 regulates YAP stability through SCF(beta-TRCP). Genes Dev 24: 72-85, 2010.

47. Huang W, Lv X, Liu C, Zha Z, Zhang H, Jiang Y, Xiong Y, Lei QY and Guan KL: The N-terminal phosphodegron targets TAZ/WWTR1 protein for SCF $\beta$-TrCP-dependent degradation in response to phosphatidylinositol 3-kinase inhibition. J Biol Chem 287: 26245-26253, 2012.

48. Liu X, Yang N, Figel SA, Wilson KE, Morrison CD, Gelman IH and Zhang J: PTPN14 interacts with and negatively regulates the oncogenic function of YAP. Oncogene 32: 1266-1273, 2013.

49. Zhao B, Li L, Lu Q, Wang LH, Liu CY, Lei Q and Guan KL: Angiomotin is a novel Hippo pathway component that inhibits YAP oncoprotein. Genes Dev 25: 51-63, 2011.

50. Oka T, Schmitt AP and Sudol M: Opposing roles of angiomotinlike-1 and zona occludens-2 on pro-apoptotic function of YAP. Oncogene 31: 128-134, 2012.

51. Hamaratoglu F, Gajewski K, Sansores-Garcia L, Morrison C, Tao $\mathrm{C}$ and Halder G: The Hippo tumor-suppressor pathway regulates apical-domain size in parallel to tissue growth. J Cell Sci 122: 2351-2359, 2009.

52. Overholtzer M, Zhang J, Smolen GA, Muir B, Li W, Sgroi DC, Deng CX, Brugge JS and Haber DA: Transforming properties of YAP, a candidate oncogene on the chromosome 11q22 amplicon. Proc Natl Acad Sci USA 103: 12405-12410, 2006.

53. Cordenonsi M, Zanconato F, Azzolin L, et al: The Hippo transducer TAZ confers cancer stem cell-related traits on breast cancer cells. Cell 147: 759-772, 2011.

54. Chan SW, Lim CJ, Guo K, Ng CP, Lee I, Hunziker W, Zeng Q and Hong W: A role for TAZ in migration, invasion, and tumorigenesis of breast cancer cells. Cancer Res 68: 2592-2598, 2008.

55. Piccolo S, Cordenonsi M and Dupont S: Molecular pathways: YAP and TAZ take center stage in organ growth and tumorigenesis. Clin Cancer Res 19: 4925-4930, 2013.

56. Wang L, Shi S, Guo Z, Zhang X, Han S, Yang A, Wen W and Zhu Q: Overexpression of YAP and TAZ is an independent predictor of prognosis in colorectal cancer and related to the proliferation and metastasis of colon cancer cells. PLoS One 8: e65539, 2013.

57. Lu L, Li Y, Kim SM, et al: Hippo signaling is a potent in vivo growth and tumor suppressor pathway in the mammalian liver. Proc Natl Acad Sci USA 107: 1437-1442, 2010.

58. Lee KP, Lee JH, Kim TS, et al: The Hippo-Salvador pathway restrains hepatic oval cell proliferation, liver size, and liver tumorigenesis. Proc Natl Acad Sci USA 107: 8248-8253, 2010

59. Avruch J, Zhou D and Bardeesy N: YAP oncogene overexpression supercharges colon cancer proliferation. Cell Cycle 11: 1090-1096, 2012

60. de Cristofaro T, Di Palma T, Ferraro A, Corrado A, Lucci V, Franco R, Fusco A and Zannini M: TAZ/WWTR1 is overexpressed in papillary thyroid carcinoma. Eur J Cancer 47: 926-933, 2011.

61. Yuen HF, McCrudden CM, Huang YH, Tham JM, Zhang X, Zeng Q, Zhang SD and Hong W: TAZ expression as a prognostic indicator in colorectal cancer. PLoS One 8: e54211, 2013. 
62. Wang Y, Dong Q, Zhang Q, Li Z, Wang E and Qiu X: Overexpression of yes-associated protein contributes to progression and poor prognosis of non-small-cell lung cancer. Cancer Sci 101: 1279-1285, 2010.

63. Xu MZ, Yao TJ, Lee NP, Ng IO, Chan YT, Zender L, Lowe SW, Poon RT and Luk JM: Yes-associated protein is an independent prognostic marker in hepatocellular carcinoma. Cancer 115: 4576-4585, 2009.

64. Xie M, Zhang L, He CS, Hou JH, Lin SX, Hu ZH, Xu F and Zhao HY: Prognostic significance of TAZ expression in resected non-small cell lung cancer. J Thorac Oncol 7: 799-807, 2012.

65. Yue G, Sun X, Gimenez-Capitan A, Shen J, Yu L, Teixido C, Guan W, Rosell R, Liu B and Wei J: TAZ is highly expressed in gastric signet ring cell carcinoma. Biomed Res Int 2014: 393064, 2014.

66. Lam-Himlin DM, Daniels JA, Gayyed MF, Dong J, Maitra A, Pan D, Montgomery EA and Anders RA: The hippo pathway in human upper gastrointestinal dysplasia and carcinoma: A novel oncogenic pathway. Int J Gastrointest Cancer 37: 103-109, 2006.

67. Zhao B, Li L, Wang L, Wang CY, Yu J and Guan KL: Cell detachment activates the Hippo pathway via cytoskeleton reorganization to induce anoikis. Genes Dev 26: 54-68, 2012.

68. Yuan M, Tomlinson V, Lara R, et al: Yes-associated protein (YAP) functions as a tumor suppressor in breast. Cell Death Differ 15: 1752-1759, 2008.

69. Danovi SA, Rossi M, Gudmundsdottir K, Yuan M, Melino G and Basu S: Yes-associated protein (YAP) is a critical mediator of c-Jun-dependent apoptosis. Cell Death Differ 15: 217-219, 2008.

70. Lai D, Ho KC, Hao Y and Yang X: Taxol resistance in breast cancer cells is mediated by the hippo pathway component TAZ and its downstream transcriptional targets Cyr61 and CTGF. Cancer Res 71: 2728-2738, 2011.

71. Liu-Chittenden Y, Huang B, Shim JS, Chen Q, Lee SJ, Anders RA, Liu JO and Pan D: Genetic and pharmacological disruption of the TEAD-YAP complex suppresses the oncogenic activity of YAP. Genes Dev 26: 1300-1305, 2012.

72. Jiao S, Wang H, Shi Z, et al: A peptide mimicking VGLL4 function acts as a YAP antagonist therapy against gastric cancer. Cancer Cell 25: 166-180, 2014.

73. Zhou Z, Hu T, Xu Z, et al: Targeting Hippo pathway by specific interruption of YAP-TEAD interaction using cyclic YAP-like peptides. FASEB J: Nov 10, 2014 (Epub ahead of print).

74. Anand R, Maksimoska J, Pagano N, Wong EY, Gimotty PA, Diamond SL, Meggers E and Marmorstein R: Toward the development of a potent and selective organoruthenium mammalian sterile 20 kinase inhibitor. J Med Chem 52: 1602-1611, 2009.

75. Rosenbluh J, Nijhawan D, Cox AG, et al: $\beta$-Catenin-driven cancers require a YAP1 transcriptional complex for survival and tumorigenesis. Cell 151: 1457-1473, 2012.

76. Frangou C, Li YW, Shen H, et al: Molecular profiling and computational network analysis of TAZ-mediated mammary tumorigenesis identifies actionable therapeutic targets. Oncotarget 5: 12166-12176, 2014.
77. Sansores-Garcia L, Bossuyt W, Wada K, Yonemura S, Tao C, Sasaki $\mathrm{H}$ and Halder G: Modulating F-actin organization induces organ growth by affecting the Hippo pathway. EMBO J 30: 2325-2335, 2011 .

78. Fernández BG, Gaspar P, Brás-Pereira C, Jezowska B, Rebelo SR and Janody F: Actin-Capping Protein and the Hippo pathway regulate F-actin and tissue growth in Drosophila. Development 138: 2337-2346, 2011

79. Wada K, Itoga K, Okano T, Yonemura S and Sasaki H: Hippo pathway regulation by cell morphology and stress fibers. Development 138: 3907-3914, 2011.

80. Thomasy SM, Morgan JT, Wood JA, Murphy CJ and Russell P: Substratum stiffness and latrunculin B modulate the gene expression of the mechanotransducers YAP and TAZ in human trabecular meshwork cells. Exp Eye Res 113: 66-73, 2013.

81. Kono K, Tamashiro DA and Alarcon VB: Inhibition of RHO-ROCK signaling enhances ICM and suppresses TE characteristics through activation of Hippo signaling in the mouse blastocyst. Dev Biol 394: 142-155, 2014

82. Miller E, Yang J, DeRan M, Wu C, Su AI, Bonamy GM, Liu J, Peters EC and $\mathrm{Wu} \mathrm{X}$ : Identification of serum-derived sphingosine-1-phosphate as a small molecule regulator of YAP. Chem Biol 19: 955-962, 2012.

83. Mo JS, Yu FX, Gong R, Brown JH and Guan KL: Regulation of the Hippo-YAP pathway by protease-activated receptors (PARs). Genes Dev 26: 2138-2143, 2012.

84. Fleming JK, Wojciak JM, Campbell MA and Huxford T: Biochemical and structural characterization of lysophosphatidic Acid binding by a humanized monoclonal antibody. J Mol Biol 408: 462-476, 2011.

85. Ponnusamy S, Selvam SP, Mehrotra S, Kawamori T, Snider AJ, Obeid LM, Shao Y, Sabbadini R and Ogretmen B: Communication between host organism and cancer cells is transduced by systemic sphingosine kinase 1/sphingosine 1-phosphate signalling to regulate tumour metastasis. EMBO Mol Med 4: 761-775, 2012.

86. Bao Y, Nakagawa K, Yang Z, Ikeda M, Withanage K, Ishigami-Yuasa M, Okuno Y, Hata S, Nishina H and Hata Y: A cell-based assay to screen stimulators of the Hippo pathway reveals the inhibitory effect of dobutamine on the YAP-dependent gene transcription. J Biochem 150: 199-208, 2011.

87. Azzolin L, Zanconato F, Bresolin S, Forcato M, Basso G, Bicciato S, Cordenonsi M and Piccolo S: Role of TAZ as mediator of Wnt signaling. Cell 151: 1443-1456, 2012.

88. Huang SM, Mishina YM, Liu S, et al: Tankyrase inhibition stabilizes axin and antagonizes Wnt signalling. Nature 461: 614-620, 2009.

89. Lau T, Chan E, Callow M, et al: A novel tankyrase smallmolecule inhibitor suppresses APC mutation-driven colorectal tumor growth. Cancer Res 73: 3132-3144, 2013.

90. Sorrentino G, Ruggeri N, Specchia V, et al: Metabolic control of YAP and TAZ by the mevalonate pathway. Nat Cell Biol 16: 357-366, 2014. 